Supplement of Biogeosciences, 16, 1401-1410, 2019

https://doi.org/10.5194/bg-16-1401-2019-supplement

(C) Author(s) 2019. This work is distributed under

the Creative Commons Attribution 4.0 License.

(c) (1)

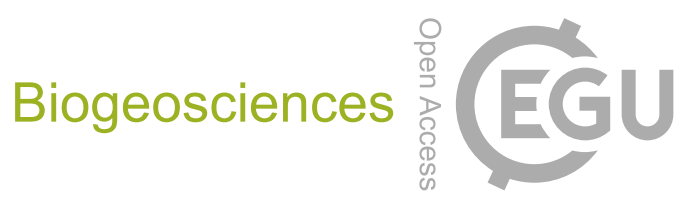

Supplement of

\title{
Multidecadal persistence of organic matter in soils: multiscale investigations down to the submicron scale
}

Suzanne Lutfalla et al.

Correspondence to: Pierre Barré (barre@biotite.ens.fr)

The copyright of individual parts of the supplement might differ from the CC BY 4.0 License. 


\section{Supplementary Materials and Methods}

\subsection{C-NEXAFS spectroscopy at the "bulk" scale}

5 The 'bulk' carbon speciation of clay sub-fractions has been investigated by Near edge X-ray absorption fine structure (NEXAFS) spectroscopy at the carbon K-edge $(270-330 \mathrm{eV})$ using the CLS beamline 11-ID-1 Spherical Grating Monochromator (SGM) (Regier et al., 2007).

Two horizontal deflecting mirrors and one vertical focusing mirror direct the light from the $45 \mathrm{~mm}$ planar undulator through the entrance slit and onto a 1700 lines/mm grating (low energy grating) which diffracts the light through a movable exit slit

10 that has been set at $25 \mu \mathrm{m}$ for the present experiments. A pair of toroidal refocusing mirrors are used to focus the beam onto the samples in the endstation chamber under a working pressure of about 10-7 Torr. A $100 \mathrm{~nm}$ thick titanium filter is used to remove the contribution of second order light. The beam spot size is approximately $1 \mathrm{~mm} \times 100 \mu \mathrm{m}$ with a flux (normalized to $100 \mathrm{~mA}$ ) greater than 1012 photons at $250 \mathrm{eV}$.

This beamline allows measuring the fluorescence yield of samples exposed to the incident beam as a function of its energy

15 with a spectral resolution of about $0.1 \mathrm{eV}$. When normalized to the incident beam (which is known through the measure of the scattering intensity from a clean gold blank), fluorescence yields are directly comparable to absorption data, especially as the SGM uses an Amptek silicon drift detector placed at exactly 90 degrees to the incident beam allowing getting rid of scattering light.

For the present study, dry particle size fractions were pressed onto gold foils (99.99 \% purity - Sigma Aldrich) which were

20 then mounted on a copper sample holder. Calibration in energy was done using a set of pure reference materials (calcium carbonate, benzoic acid and histidine (>99\% purity - Sigma Aldrich). Each spectrum reported in the present study corresponds to the average of about 50 measurements. Of note, only the first 250 nanometers of the sample surface are probed using the SGM setup. Spectra have been averaged, background subtracted and normalized using the Igor Pro software.

\subsection{STXM-based NEXAFS spectroscopy}

25 STXM-based NEXAFS data have been collected using the CLS beamline 10ID-1 (SM beamline) (Kaznatcheev et al., 2007) which works in the soft X-ray energy range (130-2500 eV) based on an elliptically polarized undulator (EPU) which provides circularly polarized light. The microscope chamber is first pumped down to $100 \mathrm{mTorr}$ after sample insertion and back-filled with He gas. A $100 \mathrm{~nm}$ thick titanium filter is used to remove the contribution of second order light. Energy calibration is done using the well-resolved 3p Rydberg peak of gaseous CO2 at $294.96 \mathrm{eV}$ for the $\mathrm{C} \mathrm{K}$-edge and using the $1 \mathrm{~s} \rightarrow \pi^{*}$ photoabsorption 
resonance of gaseous $\mathrm{N} 2$ at $400.8 \mathrm{eV}$ for the $\mathrm{N}$ K-edge. C-NEXAFS spectroscopy has been performed by collecting image stacks with a spatial resolution of 40-80 nanometers, i.e, by rastering selected areas of samples in the $x$-y directions at energy increments of $0.1 \mathrm{eV}$ over the carbon absorption energy range (270-350 eV) using the low energy grating of the 10ID-1 SM beamline. Additional image stacks have been collected at energy increments of $1 \mathrm{eV}$ over the $270-450 \mathrm{eV}$ energy range to

5 identify the clay minerals associated with the measured SOC particles. Indeed, the presence of potassium and calcium can be seen as the signature of clay minerals: they are present in the interlayer and surface sites of illites and swelling clays, respectively. Stack measurements have been performed with a dwell time of one millisecond or less per pixel to prevent irradiation damage following the procedures for X-ray microscopy studies of radiation sensitive samples recommended in the literature (Wang et al., 2007). Alignment of images of stacks and extraction of NEXAFS spectra have been done using the

10 aXis2000 software (ver2.1n). Spectral peak positions, intensities and widths have been determined using the Athena software package (Ravel and Newville, 2005). The C-NEXAFS spectra shown in the present contribution correspond to homogeneous organic-rich areas of several hundreds of square nanometers. Extensive databases of reference C-NEXAFS spectra are available in the literature (Solomon et al., 2009).

\subsection{C-NEXAFS data deconvolution procedure}

15 To obtain a more quantitative insight on the evolution of the molecular signatures of the investigated experimental samples with increasing bare fallow duration and to be able to compare the spectra, we performed a three steps deconvolution: i) background subtraction, ii) normalization to the carbon amount and iii) fit of Gaussian functions. After a pre-edge background subtraction (270-282 eV), normalization to the amount of carbon (density* thickness of the sample) is then required. This is usually performed by a post-edge linear regression (Bernard et al., 2010; Braun et al., 2006). However, most of the published

20 data (including those from our own previous works), are acquired for practical reasons only up to $320 \mathrm{or} 340 \mathrm{eV}$. At these energies, EXAFS oscillations can still be present and therefore bias the post-edge normalization which in turn will also bias the relative peak height. Normalization could only be safely done if the spectra were acquired up to at least $350 \mathrm{eV}$. To circumvent this normalization issue for datasets not acquired over such a long energy range, we adopted an alternative approach. We first compared reference spectra from chemically different materials (amino acids, hexamethylenetetramine,

25 bacterias, complex macromolecular OM from chondrites) acquired up to $390 \mathrm{eV}$. We applied the classical post-edge normalization using the Athena software (between 370 and $390 \mathrm{eV}$ ) and realized that the area of the spectra between $280 \mathrm{eV}$ and $291.5 \mathrm{eV}$-i.e. near the ionization energy of carbon in OM- was constant (with 10\% standard deviation). Therefore, for the present dataset, instead of using the potentially misleading post-edge signal, we normalized each spectrum to its own area, calculated up to $291.5 \mathrm{eV}$. This treatment ensures that the carbon amount is properly taken into account and that spectra are

30 chemically consistent, i.e. that, by construction, a spectrum showing a more prominent absorption than others at a given energy shows a less intense absorption at the energy of the other functional groups.

After normalization, spectra were fitted using gaussian functions, placed at a fixed positions corresponding to known functional groups (Bernard et al., 2012; Le Guillou et al., 2014), and adapted from a previously described (Bernard et al., 2010) based on 
previously published recommendations (Braun et al., 2006; Carravetta et al., 1998). We upgraded the positions and widths of the gaussians to better take in into account the various peak assignments described in the literature (De Gregorio et al., 2010; Dhez et al., 2003; Urquhart et al., 1999). We used a full-width at half maximum of $0.6 \mathrm{eV}$ below $295 \mathrm{eV}$ and of $2 \mathrm{eV}$ above, in order to take into account the multiple scattering effects.

5 The following positions and assignments were considered: 284.4, quinones; $285 \mathrm{eV}, 285.4 \mathrm{eV}$, aromatic; $285.8 \mathrm{eV}$, imines; $286.2 \mathrm{eV}, 286.6 \mathrm{eV}, 287.1 \mathrm{eV}$, carbonyls; $287.7 \mathrm{eV}$, aliphatics; $288.2 \mathrm{eV}$, amides; $288.6 \mathrm{eV}$, carboxylic; $289.1 \mathrm{eV}$, aldehydes; $289.4 \mathrm{eV}$, hydroxyls; $289.9 \mathrm{eV}$, aliphatics; $290.3 \mathrm{eV}$, carbonates. Additional bands at 290.8, 291.2, 291.7, 292.2, 292.7, 293.4, 294, 295, 297.5, 300, 302.5, 305, 307.5, 310 and $312.5 \mathrm{eV}$ are introduced as well. Most of the latter Gaussian functions do not account for the presence of known functional groups, but rather for broad spectral features corresponding to highly delocalized

10 excited states, sometimes referred to as $1 \mathrm{~s}-\sigma^{*}$ virtual state transitions, or for the overlapping contribution of Feshbach resonances (Stöhr, 1991). The extracted gaussian heights were then used to compare the relative abundances and the evolution of the various functional groups. 


\section{Supplementary Figures}

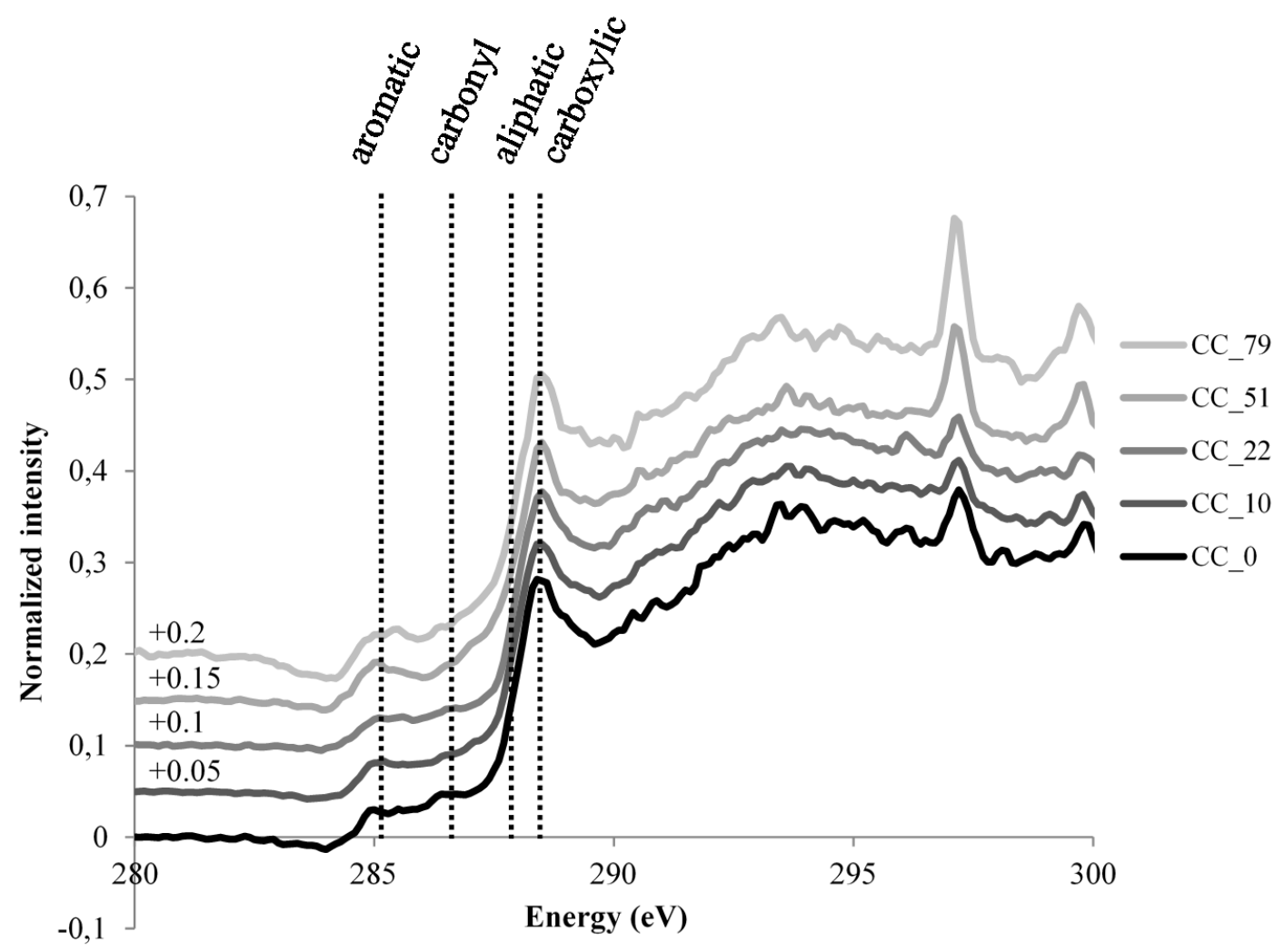

Figure S1: Normalized NEXAFS spectra for the coarse clay subfraction (labeled CC) at all dates: from the initial sampling date (year 0 of bare fallow, bottom spectrum, darkest color) to the final sampling date (year 79 of bare fallow, upper spectrum, lightest color). The four main spectral features are labeled according to the corresponding chemical moieties. 


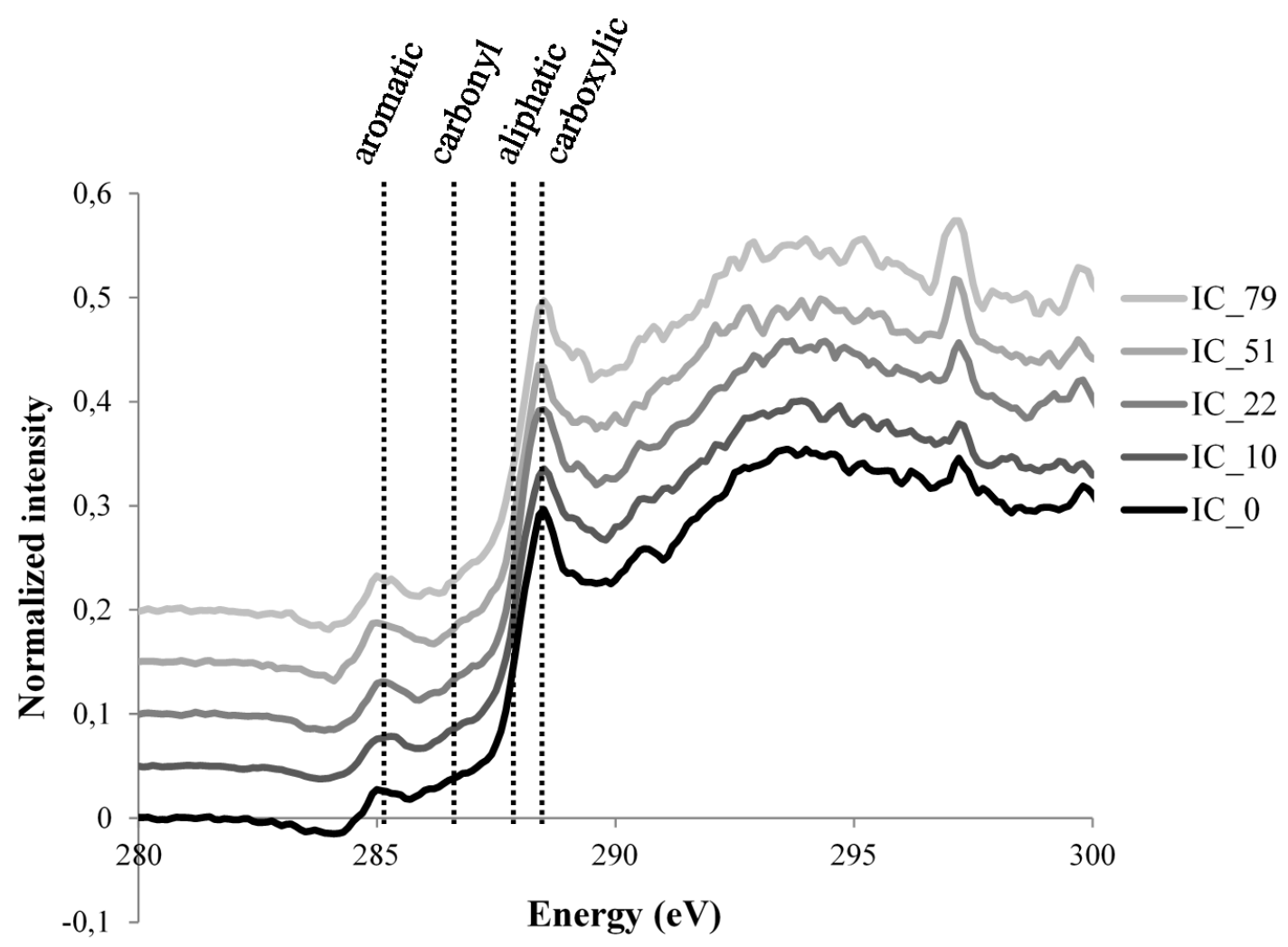

Figure S2: Normalized NEXAFS spectra for the intermediate sub-fraction (labeled IC) at all dates: from the initial sampling date (year 0 of bare fallow, bottom spectrum, darkest color) to the final sampling date (year 79 of bare fallow, upper spectrum, lightest color). The four main spectral features are labeled according to the corresponding chemical moieties. 

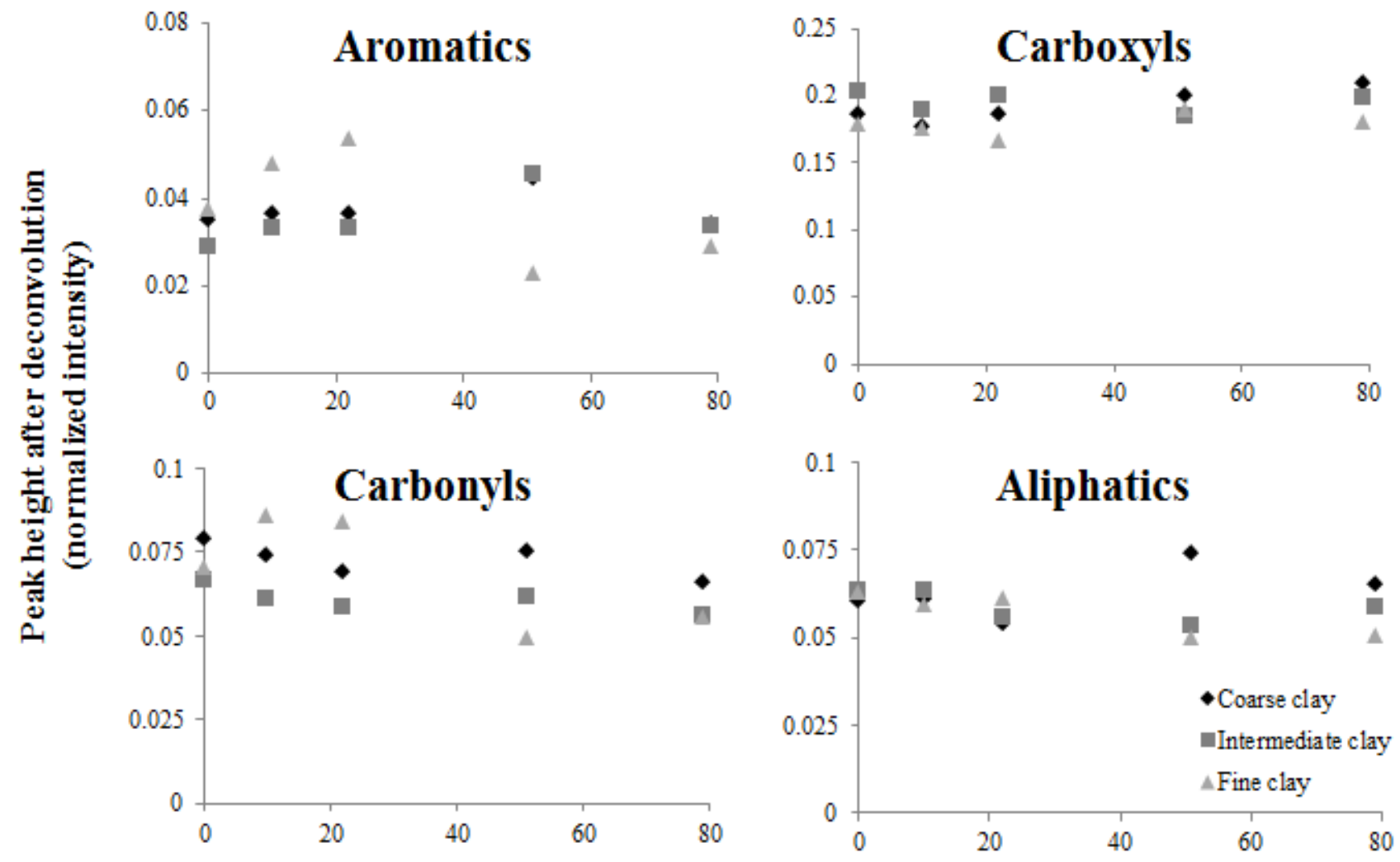

\section{Bare fallow duration (years)}

Figure S3: Evolution of the peak heights with time for the four main functional groups (aromatics, carbonyls, carboxylics and aliphatics), for all clay subfractions. Data obtained by deconvoluting the spectra obtained by 'bulk' NEXAFS.

The relative contents of each functional groups, as seen by peak heights, are similar for all sub-fractions. For each sub-fraction, aromatic contents are highly variable with time yet no significant trend was detected. Contents in carbonyl groups do not

5 significantly evolve with time in the sub-fractions, aliphatic and carboxylic groups do not significantly evolve with time except for the carboyxlics moeities contained in FC which are increasing with time and the aliphatics in the $\mathrm{CC}$ sub-fraction which are decreasing. 

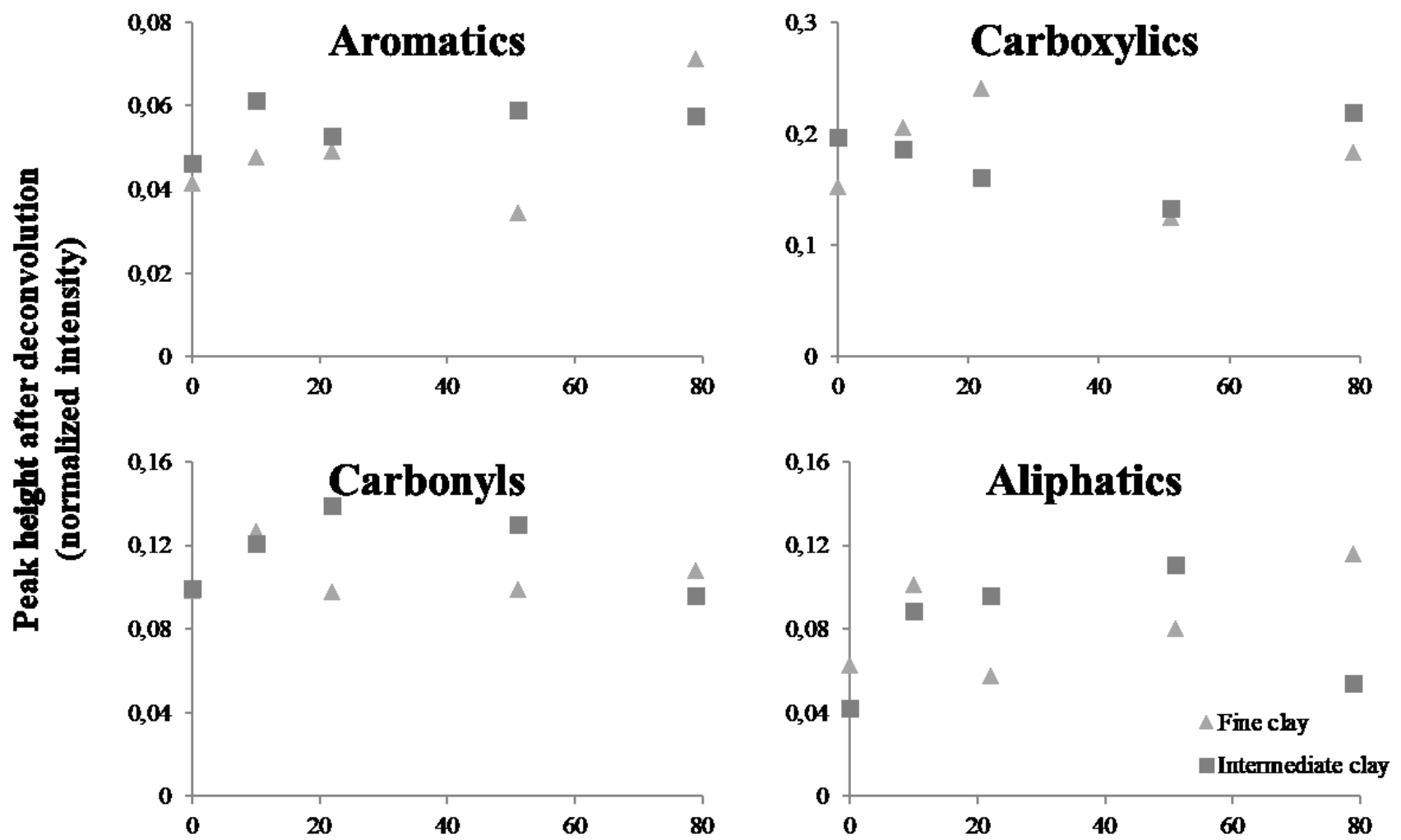

Bare fallow duration (years)

Figure S4: Evolution of the peak heights with time for the four main functional groups (aromatics, carbonyls, carboxylics and aliphatics), for fine and intermediate clay subfractions. Data obtained by deconvoluting the spectra obtained by data treatment of the STXM-NEXAFS hyperspectral maps. 


\section{References}

Bernard, S., Beyssac, O., Benzerara, K., Findling, N., Tzvetkov, G. and Brown, G. E.: XANES, Raman and XRD study of anthracene-based cokes and saccharose-based chars submitted to high-temperature pyrolysis, Carbon, 48(9), 2506-2516, doi:10.1016/j.carbon.2010.03.024, 2010.

5 Braun, A., Huggins, F. E., Kelly, K. E., Mun, B. S., Ehrlich, S. N. and Huffman, G. P.: Impact of ferrocene on the structure of diesel exhaust soot as probed with wide-angle X-ray scattering and C(1s) NEXAFS spectroscopy, Carbon, 44(14), 2904-2911, doi:10.1016/j.carbon.2006.05.051, 2006.

Carravetta, V., Polzonetti, G., Iucci, G., Russo, M. V., Paolucci, G. and Barnaba, M.: High resolution NEXAFS spectroscopy study of gas-phase phenylacetylene: experiment and theory, Chem. Phys. Lett., 288(1), 37-46, doi:10.1016/S0009-

$10 \quad 2614(98) 00143-2,1998$.

De Gregorio, B. T., Stroud, R. M., Nittler, L. R., Alexander, C. M. O., Kilcoyne, A. L. D. and Zega, T. J.: Isotopic anomalies in organic nanoglobules from Comet 81P/Wild 2: Comparison to Murchison nanoglobules and isotopic anomalies induced in terrestrial organics by electron irradiation, Geochim. Cosmochim. Acta, 74(15), 4454-4470, doi:10.1016/j.gca.2010.05.010, 2010.

15 Dhez, O., Ade, H. and Urquhart, S. G.: Calibrated NEXAFS spectra of some common polymers, J. Electron Spectrosc. Relat. Phenom., 128(1), 85-96, doi:10.1016/S0368-2048(02)00237-2, 2003.

Kaznatcheev, K. V., Karunakaran, C., Lanke, U. D., Urquhart, S. G., Obst, M. and Hitchcock, A. P.: Soft X-ray spectromicroscopy beamline at the CLS: Commissioning results, Nucl. Instrum. Methods Phys. Res. Sect. Accel. Spectrometers Detect. Assoc. Equip., 582(1), 96-99, doi:10.1016/j.nima.2007.08.083, 2007.

20 Ravel, B. and Newville, M.: ATHENA, ARTEMIS, HEPHAESTUS: data analysis for X-ray absorption spectroscopy using IFEFFIT, J. Synchrotron Radiat., 12(4), 537-541, doi:10.1107/S0909049505012719, 2005.

Regier, T., Krochak, J., Sham, T. K., Hu, Y. F., Thompson, J. and Blyth, R. I. R.: Performance and capabilities of the Canadian Dragon: The SGM beamline at the Canadian Light Source, Nucl. Instrum. Methods Phys. Res. Sect. Accel. Spectrometers Detect. Assoc. Equip., 582(1), 93-95, doi:10.1016/j.nima.2007.08.071, 2007.

25 Solomon, D., Lehmann, J., Kinyangi, J., Liang, B., Heymann, K., Dathe, L., Hanley, K., Wirick, S. and Jacobsen, C.: Carbon (1s) NEXAFS Spectroscopy of Biogeochemically Relevant Reference Organic Compounds All rights reserved. No part of this periodical may be reproduced or transmitted in any form or by any means, electronic or mechanical, including photocopying, recording, or any information storage and retrieval system, without permission in writing from the publisher. Permission for printing and for reprinting the material contained herein has been obtained by the publisher., Soil Sci. Soc. Am. J., 73(6),

30 1817-1830, doi:10.2136/sssaj2008.0228, 2009.

Stöhr, J.: NEXAFS Spectroscopy, Springer, New York., 1991. 
Urquhart, S. G., Hitchcock, A. P., Smith, A. P., Ade, H. W., Lidy, W., Rightor, E. G. and Mitchell, G. E.: NEXAFS spectromicroscopy of polymers: overview and quantitative analysis of polyurethane polymers, J. Electron Spectrosc. Relat. Phenom., 100(1), 119-135, doi:10.1016/S0368-2048(99)00043-2, 1999.

Wang, C.-H., Chien, C.-C., Yu, Y.-L., Liu, C.-J., Lee, C.-F., Chen, C.-H., Hwu, Y., Yang, C.-S., Je, J.-H. and Margaritondo,

5 G.: Structural properties of 'naked' gold nanoparticles formed by synchrotron X-ray irradiation, J. Synchrotron Radiat., 14(6), 477-482, doi:10.1107/S0909049507044743, 2007. 